\title{
Programa de prevenção para manejo de estresse e Síndrome de Burnout para bombeiros: Relato de experiência de uma intervenção
}

Lucia Petrucci de Melo. Sheraton Hotel Porto Alegre.

Mary Sandra Carlotto. Universidade do Vale do Rio dos Sinos.

\section{Resumo}

Este artigo relata a experiência de uma intervenção cujo objetivo foi apresentar uma proposta de programa de prevenção para manejo de estresse e Síndrome de Burnout a profissionais bombeiros. O programa, com três meses de duração, periodicidade quinzenal foi estruturado em cinco sessões. São apresentados os objetivos de trabalho de cada sessão, bem como os procedimentos e técnicas utilizados. O programa possibilitou aos participantes lidarem melhor com os estressores laborais. Estudos dessa modalidade de intervenção permitem aos profissionais desenvolverem novas habilidades e que estas sejam conectadas com o seu trabalho.

Palavras-chave: burnout; estresse profissional; intervenção; bombeiros.

\begin{abstract}
Stress management and Burnout Syndrome prevention program for firefighters: Report of an intervention experience. This article describes the experience of an intervention that aimed to present a stress and Burnout management prevention program for firefighters. The program, with three months' duration on a fortnightly basis, was divided into five sessions. This report presents the work objectives of each session, as well as the procedures and techniques used. The program allowed participants to cope better with work stressors. Intervention studies allow professionals develop new skills and connect them with their work.
\end{abstract}

Keywords: burnout; professional stress; intervention; firefighters.

\section{Resumen}

Programa de prevención para el manejo de estrés e Síndrome de Burnout para bomberos: Informe de una experiencia de intervención. Este artículo reporta la experiencia de una intervención que tuvo como objetivo presentar una propuesta de programa de prevención para el manejo del estrés y del síndrome de Burnout entre profesionales bomberos. El programa con tres meses de duración y periodicidad quincenal fue estructurado en cinco sesiones. Este informe presenta los objetivos de trabajo de cada sesión así como los procedimientos y técnicas utilizados. El programa posibilitó a los participantes que pudieran lidiar mejor con los estresores laborales. Estudios de intervención posibilitan a los profesionales que desarrollen nuevas habilidades y que esas sean conectadas a su trabajo.

Palabras clave: burnout; estrés profesional; intervención; bomberos. 
A síndrome de burnout (SB) tem sido estudada desde a década de 1970, mas ainda hoje reflete um importante desafio na vida laboral. É entendida como um fenômeno psicossocial que ocorre como consequência da exposição de longo prazo às condições de trabalho adversas, como excessiva pressão, conflitos, falta de recompensas e reconhecimento (Harrison, 1999; Maslach, Schaufeli, \& Leiter, 2001; Salanova \& Llorens, 2008). Trata-se do resultado das progressivas tentativas malsucedidas de lidar com os estressores presentes no contexto de trabalho (Byrne, 1993; Leiter, Bakker, \& Maslach, 2014; Maslach, Schaufeli, \& Leiter, 2001;).

A definição mais aceita e empregada na literatura internacional no tocante à SB é a fundamentada na perspectiva psicossocial de Maslach e Jackson (1981), que a define como um fenômeno estruturado em três dimensões: 1) Exaustão emocional, entendida como a ausência de energia e sentimento de esgotamento dos recursos emocionais do sujeito, os quais podem somar-se à frustração e tensão com a percepção de que o sujeito já não tem energia e disposição para atender às demandas de seu trabaIho; 2) Despersonalização ou desumanização, caracterizada por sentimentos negativos e insensibilidade emocional para com as pessoas com as quais o sujeito trabalha, passando a tratar clientes, colegas e organização de maneira distante e impessoal; e 3) Baixa realização profissional, que trata de sentimentos de incompetência e falta de realização e produtividade no trabalho, o trabalhador demonstra insatisfação com seu desenvolvimento profissional e seu autoconceito e autoestima baixam, assim, experimentando sentimento de declínio em seu êxito profissional, bem como em seus relacionamentos com clientes, pacientes e mesmo com colegas.

As pessoas com Burnout desenvolvem sentimentos de desilusão e declínio da motivação, assim, ocasionando consequências negativas tanto para os trabalhadores como para as equipes de trabalho e para os resultados organizacionais (Carlotto, 2012; Cebrià-Andreu, 2005; Gil-Monte, 2005; Salanova \& Llorens, 2008). O Burnout é gerador de importantes perdas de recursos humanos e econômicos, podendo levar os profissionais ao afastamento laboral, de curto e médio prazo, por ansiedade, depressão, transtornos do estresse, transtornos psicossomáticos e mesmo ideação suicida (Trigo, Teng, \& Hallak, 2007).
Apesar do seu carácter universal, ainda são poucas as pesquisas dedicadas a avaliar intervenções destinadas a reduzir o Burnout (Halbesleben \& Buckley, 2004; Maslach et al., 2001). A intervenção para a SB deve ser entendida como uma ação conjunta entre indivíduo e organização, focalizada tanto na esfera microssocial do trabalhador, com sua atividade e suas relações interpessoais, como na ampla gama de fatores macro-organizacionais que determinam aspectos que constituem a cultura organizacional e social na qual o sujeito exerce sua atividade profissional (Carlotto \& Gobbi, 1999). Ao propor programas de intervenção é fundamental que se esteja atento primordialmente aos aspectos de prevenção e aos sinais precoces de desenvolvimento da SB (Maslach \& Leiter, 2008).

Os programas comumente utilizados para prevenir ou reduzir o desenvolvimento da SB são direcionados aos trabalhadores, à organização ou, ainda, mistos - quando abrangem aspectos individuais do trabalhador e da organização (Awa, Plaumann, \& Walter, 2010; Halbesleben, Osburn, \& Mumford, 2006; Van der Klink, Blonk, Schene, \& Van Dijk, 2001). Quando direcionados à organização, os programas enfocam mudanças no ambiente organizacional, como procedimentos de trabalho, reestruturação de tarefas, avaliação do trabalho e supervisão, que visam diminuir a demanda de trabalho, aumentando o controle sobre o mesmo ou o nível de participação nas tomadas de decisões (Awa, Plaumann, \& Walter, 2010). Quando direcionados aos indivíduos, os programas geralmente visam ampliar e qualificar as habilidades pessoais do trabalhador, suas estratégias de enfrentamento, ampliar o apoio social e emocional, aumentar a competência profissional estimulando a formação continuada, reformular a execução do trabalho buscando novas formas de realização, estimular atividades extralaborais na forma de prática de exercícios físicos, de esportes, de relaxamento e busca de algum hobby particular, realizar momentos de descansos (pausas) durante o trabalho e projetar objetivos reais e factíveis e emocional (Abalo \& Roger, 1998; Awa, Plaumann, \& Walter, 2010; Gunasingam, Burns, Edwards, Dinh, \& Walton, 2015; Murphy, 1996; Salanova \& Llorens, 2008).

Os programas com foco no trabalhador têm sido os mais frequentemente adotados. Estudo de revisão sistemática identificou que $82 \%$ de todas as intervenções em âmbito individual levaram a uma redução 
significativa no desgaste ou mudanças positivas nos fatores de risco de Burnout (Awa, Plaumann, \& Walter, 2010). Estudo com enfermeiros oncológicos portugueses avaliou o impacto da intervenção para atenuar o Burnout e revelou um aumento das competências dos profissionais para lidar com as variáveis indutoras da síndrome, constituindo-se em uma medida adequada de prevenção (Soares \& Rocha, 2010). Trabalho realizado com profissionais holandeses que cuidam de pessoas com deficiência mental avaliou a eficácia de um programa de intervenção em Burnout, com duração de cinco semanas, e mostrou que a exaustão emocional pode realmente ser reduzida, principalmente pela capacitação de profissionais para usar habilidades de enfrentamento, tais como técnicas de relaxamento, reestruturação cognitiva e habilidades sociais (Dierendonck, Schaufeli, \& Buunk, 1998). No Brasil, Murta e Trocolli (2007) desenvolveram uma intervenção psicoeducativa com bombeiros, abordando temas como causas e efeitos do estresse, fatores de risco e de proteção e estratégias de enfrentamento, estabelecimento de metas, manejo de tempo, habilidades sociais, solução de problemas, reestruturação de crenças irracionais, relaxamento e prevenção de recaída. A avaliação da intervenção revelou redução nos níveis de estresse e sintomas somáticos e aumento da autoeficácia e pressão arterial diastólica.

Em relação à duração desses programas, observa-se que as intervenções direcionadas aos trabalhadores podem variar de dois dias a três meses (Awa, Plaumann, \& Walter, 2010). Em experimento que explorou abordagens de curto e longo prazo para medir a mudança gerada no comportamento dos participantes em relação ao Burnout, evidenciou-se que os profissionais que participaram do programa de seis semanas apresentaram reduções temporárias de Burnout, enquanto que os profissionais participantes da intervenção de 17 meses revelaram decréscimos consistentes em todas as dimensões de Burnout (Rowe, 2000).

Atualmente, o Burnout já deixou de ser uma temática de interesse e investigação acadêmica e tem cada vez mais se consolidado como uma importante prática e de intervenção profissional que envolve trabalhadores e gestores (Leiter et al., 2014). Desta forma, considerando a escassez de intervenções direcionadas à redução da SB, este relato de experiência de intervenção tem por objetivo apresentar uma proposta de programa de prevenção para manejo de estresse e SB no tocante a profissionais bombeiros, desse modo, contribuindo para a discussão de modelos e procedimentos de intervenção.

\section{Método}

\section{Participantes}

A intervenção foi oferecida ao $1^{\circ}$. Comando do Corpo de Bombeiros do estado do Rio Grande do Sul - Brasil, composta por 336 profissionais, e transcorreu no ano de 2014. Cinco bombeiros se inscreveram voluntariamente e participaram integralmente do programa. A participação foi voluntária, uma vez que a obrigatoriedade pode gerar reações de hostilidade e rejeição à intervenção, assim, aumentando as chances de desistência e abandono (Murta, Laros, \& Troccoli, 2005).

O grupo foi formado exclusivamente por profissionais do sexo masculino, com idades entre $37 \mathrm{e}$ 50 anos, casados, com filhos e, em sua maioria, com escolaridade em nível de ensino médio completo (75\%). O tempo médio de atuação dos profissionais como bombeiro era de 23 anos ( $D P=7,77$ ), ocupando o posto de Sargento $(87,5 \%)$ e Soldado $(12,5 \%)$, e combatente de fogo $(87,5 \%)$, atendendo uma média de sete ocorrências semanais ( $D P=5,7$ ).

\section{Procedimentos de intervenção}

Mediante autorização da instituição para a realização da intervenção, o programa foi apresentado ao grupo de profissionais para que os interessados se inscrevessem voluntariamente como participantes. Os procedimentos éticos seguiram a Resolução 446/12 do Conselho Nacional de Saúde (Ministério da Saúde, 2012), referente à pesquisa com seres humanos, sob Protocolo $n^{\circ} 512.521$.

O programa, com foco no trabalhador, foi estruturado em cinco sessões, com periodicidade quinzenal e durabilidade de três meses. Os encontros ocorreram no auditório da instituição visando ao fácil acesso, sigilo e à privacidade dos participantes. As sessões foram realizadas no turno da noite, após o expediente de trabalho, e tiveram duas horas de duração cada. A síntese da temática, objetivo e técnica de cada sessão é apresentada no quadro 1. A condução das sessões foi desenvolvida pela primeira autora do estudo e contou com a presença de um observador, aluno bolsista de Iniciação Científica do curso de Psicologia, responsável por registrar os eventos relevantes ocorridos em cada sessão para compor o diário de campo. 
Programa de prevenção para manejo de estresse e Síndrome de Burnout para bombeiros: Relato de experiência de uma intervenção

\begin{tabular}{|c|c|c|c|}
\hline Módulo & Temática & Objetivo & Técnica \\
\hline 1 & $\begin{array}{l}\text { Contrato de trabalho e } \\
\text { autodiagnóstico }\end{array}$ & $\begin{array}{l}\text { Realizar o contrato de trabalho, apresentação dos participan- } \\
\text { tes e da coordenadora. Informar sobre a SB, suas principais } \\
\text { características, sintomas e consequências. Estimular o } \\
\text { reconhecimento e a existência dos estressores presentes na } \\
\text { profissão do bombeiro. }\end{array}$ & $\begin{array}{l}\text { Exposição teórica. } \\
\text { Atividade prática de reconhecimento dos estres- } \\
\text { sores laborais. } \\
\text { Discussão livre. } \\
\text { Tarefa de casa: escreva sua própria narrativa. }\end{array}$ \\
\hline 2 & $\begin{array}{l}\text { Estratégias de enfrentamento } \\
\text { - coping }\end{array}$ & $\begin{array}{l}\text { Reconhecer as estratégias saudáveis para lidar com os estres- } \\
\text { sores específicos da profissão. }\end{array}$ & $\begin{array}{l}\text { Exposição teórica. } \\
\text { Atividade prática de encenação dos estressores } \\
\text { da profissão e relação com as estratégias de } \\
\text { coping. } \\
\text { Discussão livre. } \\
\text { Tarefa de casa: o que tem no seu prato? }\end{array}$ \\
\hline 3 & Manejo de estresse & $\begin{array}{l}\text { Construir o plano de gerenciamento do estresse, como fer- } \\
\text { ramenta de apoio para auxiliar o profissional no manejo e } \\
\text { prevenção do estresse. }\end{array}$ & $\begin{array}{l}\text { Exposição teórica. } \\
\text { Atividade prática de construção do plano de } \\
\text { gerenciamento do estresse. } \\
\text { Discussão livre. } \\
\text { Tarefa de casa: reduza o estresse. Aprenda a dizer } \\
\text { não (ou sim)! }\end{array}$ \\
\hline 4 & Fadiga da compaixão & $\begin{array}{l}\text { Desenvolver habilidades para a promoção da saúde mental } \\
\text { do profissional perante situações de sofrimento emocional } \\
\text { ao qual estão expostos na sua profissão. }\end{array}$ & $\begin{array}{l}\text { Exposição teórica. } \\
\text { Atividade prática de comunicação. } \\
\text { Discussão livre. } \\
\text { Tarefa de casa: fechamento do plano de gerencia- } \\
\text { mento do estresse. }\end{array}$ \\
\hline 5 & $\begin{array}{l}\text { Treinamento de habilidades } \\
\text { sociais, fechamento e } \\
\text { avaliação }\end{array}$ & $\begin{array}{l}\text { Instruir, modelar, reforçar, ensaiar e generalizar condutas } \\
\text { alternativas frente às demandas da vida laboral-profissional } \\
\text { e pessoal, principalmente aquelas relacionadas à compe- } \\
\text { tência interpessoal. Realizar o fechamento do trabalho e a } \\
\text { integração temática de cada encontro. Realizar a avaliação } \\
\text { final da intervenção. }\end{array}$ & $\begin{array}{l}\text { Exposição teórica. } \\
\text { Atividade prática de ensaio do comportamento. } \\
\text { Discussão livre. Avaliação final. }\end{array}$ \\
\hline
\end{tabular}

Figura 1. Quadro de Caracterização da Proposta de Intervenção.

\section{Relato da experiência}

As sessões serão descritas uma a uma, relatando os aspectos referentes ao objetivo, procedimentos e técnicas utilizadas em cada encontro da intervenção. 0 primeiro encontro destinou-se à abertura do programa de intervenção e teve por objetivo realizar o contrato de trabalho, informar os participantes sobre a SB, suas principais características, sintomas e consequências e estimular o autoconhecimento por meio do reconhecimento dos principais estressores presentes na profissão do bombeiro.

A sessão iniciou com uma apresentação expositiva dos objetivos da intervenção de prevenção para manejo de estresse e SB. Após exposição da coordenadora, os participantes fizeram uma apresentação individual e laboral e expuseram suas expectativas em relação ao trabalho. As expectativas estavam relacionadas à qualidade de vida, ao autoconhecimento, ao relacionamento com colegas e chefias, à oportunidade de aprendizado e ao aproveitamento das horas de treinamento com perspectivas de mudança de cargo.

A partir da exposição foi possível realizar esclarecimentos em relação ao que seria oferecido na intervenção, bem como a exposição do acordo de trabalho, em que foi enfatizado tratar-se de uma atividade voluntária e que os participantes poderiam deixar de participar a qualquer momento. Foi reforçado o compromisso com o sigilo e resguardo das informações geradas nos encontros. Apresentaram-se as datas, horários, temas e tempo de duração de cada encontro para que os participantes pudessem assegurar sua continuidade. Com as informações oferecidas neste primeiro momento, os participantes interessados assinaram o Termo de Consentimento Livre e Esclarecido (TCLE).

Antes de abordar a temática da primeira sessão, foi executada uma sensibilização e proposta uma atividade de reconhecimento dos principais estressores da profissão de bombeiro como a sobrecarga de trabaIho, a carga horária e sistema de turnos, a exposição a substâncias perigosas, o contato direto com a morte de jovens e crianças e acidentes envolvendo colegas de trabalho. Com base no material construído pelo grupo, foi realizada a exposição e discussão do entendimento da SB e sua relação com a atividade profissional do bombeiro. Foram, então, apresentadas as possíveis consequências da SB ao indivíduo no âmbito físico, psíquico, emocional e comportamental. 
No fechamento do encontro, repassaram-se os principais pontos tratados e foi entregue, aos participantes, uma tarefa de casa, para ser discutida no próximo encontro. As tarefas de casa constituem uma maneira de as habilidades aprendidas na sessão serem praticadas no ambiente real, ou seja, serem generalizadas à vida diária do indivíduo. Elas são benéficas para o acesso aos comportamentos privados e para um maior autocontrole e eficácia do treinamento (Caballo, 2010).

A atividade proposta para o fechamento da primeira sessão chama-se Escreva sua própria narrativa (Mathieu, 2012) consiste em o profissional escrever uma narrativa identificando os principais estressores de seu trabalho, bem como descrever as estratégias geralmente adotadas para o seu manejo. A identificação dos principais estressores da profissão é importante para o processo de reconhecimento de estratégias de enfrentamento adequadas para lidar com os mesmos (Brock \& Grady, 2000). Com essa conscientização e sensibilização, o indivíduo é motivado a engajar-se em atividades de autocuidado a fim de promover o seu bem-estar físico e emocional. $\mathrm{O}$ autocuidado é um processo cognitivo, afetivo e comportamental desenvolvido ao longo da vida, que se consolida quando a pessoa se compromete a assumir responsabilidade pessoal pela condução da sua própria vida, em direção à conquista da integralidade nas relações consigo e com o mundo no qual se encontra inserida (Neves \& Wink, 2007).

O segundo encontro teve por objetivo trabalhar a temática Estratégias de Enfrentamento - coping. $\mathrm{O}$ coping é entendido como um conjunto de respostas intencionais, cognitivas e comportamentais, utilizadas pelo sujeito com o propósito de lidar com demandas específicas oriundas de situações estressantes, que podem ser aprendidas, utilizadas e descartadas (Lazarus \& Folkman, 1984). No modelo proposto por Folkman e Lazarus (1980), as respostas ou estratégias de coping são divididas em duas categorias: foco no problema e foco na emoção. O coping focalizado no problema constitui-se no esforço despendido pelo sujeito para atuar na situação que deu origem ao estresse, desse modo, tentando mudá-la. A função desta estratégia é alterar o problema existente na relação entre a pessoa e o ambiente que está causando a tensão. O coping focalizado na emoção é compreendido como um esforço para regular o estado emocional que é associado ao estresse e reduzir a sensação física desagradável que é gerada. Esses esforços são dirigidos a um nível somático e/ou a um nível de sentimentos, tendo por consequência a alteração do estado emocional do indivíduo.

Antes de iniciar as atividades previstas, a coordenadora repassou com o grupo a tarefa de casa a fim de discutir sua realização. Os participantes relataram a experiência de escrever sua narrativa e enfatizaram a oportunidade de refletir acerca dos principais estressores de seu trabalho e a forma como lidam com essas situações no seu dia a dia. A partir do relato das narrativas, construiu-se uma vinculação com o tema desta sessão na medida em que a proposta era analisar a forma de lidar com os estressores ocupacionais. Nesse sentido, foi apresentado, então, ao grupo, o conceito teórico de coping (Lazarus \& Folkman, 1984) enfatizando a diferença entre estratégias focadas no problema e estratégias centradas nas emoções enfatizando-se que a utilização de estratégias focadas no problema possuem maior capacidade de prevenir a SB (Gil-Monte, 2005; Mirvis, Graney, Ingram, Tang, \& Kilpatrick, 2006).

Após a exposição conceitual, os participantes realizaram uma atividade organizados em uma dupla e um trio, podendo escolher livremente com quem desejassem fazê-la. Cada subgrupo conversou sobre a narrativa construída na atividade de casa, apresentando os principais pontos que lhe geram estresse no trabalho e quais as estratégias que comumente utilizam para enfrentá-los, buscando relacioná-las com as classificações apresentadas anteriormente. Após, o grupo escolheu um fator estressor da profissão para que fosse debatido considerando as estratégias de enfrentamento, identificando os benefícios gerados pelas estratégias focadas no problema, consideradas mais saudáveis. Essa atividade teve por objetivo incentivar a troca de experiências e proporcionar maior associação da prática aos conteúdos conceituais expostos, assim, permitindo que os participantes pudessem aprender uns com os outros.

Uma vez trabalhadas as estratégias de enfrentamento, buscou-se associá-las com ações comportamentais que possibilitam a prevenção da SB. Ainda que seja difícil evitar o estresse por completo, o indivíduo pode adotar uma série de precauções para prevenir seus níveis mais elevados, tais como o desenvolvimento de relações saudáveis no trabalho com colegas e superiores, desenvolvimento de um plano pessoal para alívio do estresse, avaliação de seu estilo de vida, busca por aconselhamento profissional, aprender a pedir ajuda, desenvolver o humor, ouvir música, ser criativo, fazer bom uso de seu tempo de descanso, praticar exercícios, estudar e escrever (Florio, 2010). 
Após a exposição e debate das boas práticas de combate ao Burnout, deu-se o fechamento do encontro com a revisão dos pontos discutidos e apresentação da tarefa de casa, que consistiu em incentivar os participantes a identificarem as principais situações estressantes de seu dia a dia e explorarem as melhores maneiras de lidar com elas. Para tanto, utilizou-se a atividade individual chamada $O$ que tem no seu prato? (Mathieu, 2012).

O terceiro encontro objetivou desenvolver habilidades para o manejo de estresse. A sessão iniciou com a retomada da atividade de casa. Os participantes relataram a surpresa que sentiram em listar todas as atividades que realizam em seu dia a dia, bem como de que maneira essa tarefa possibilitou refletir sobre a natureza das atividades que estão realizando, quais as que mais são geradoras de estresse e o que estão fazendo para manejá-lo.

Com base nas reflexões dos participantes, expôs-se ao grupo o entendimento de estresse, estresse ocupacional e a diferença entre estresse e Burnout. 0 estresse tem um caráter, geralmente, episódico, que, quando prolongado, leva a sintomas crônicos e ao Burnout, assim, o estresse e Burnout não podem ser diferenciados com base em seus sintomas, mas sim em seu processo (Kyriacou \& Sutcliffe, 1978; Maslach \& Schaufeli, 1993).

Apresentado e debatido o entendimento conceitual, o encontro foi dedicado à realização da atividade de construção do plano de gerenciamento de estresse. Essa atividade é desenvolvida individualmente e orientada pela coordenação do encontro. O plano é dividido da seguinte forma: Respiração: ensinamento de exercício respiratório para alívio da ansiedade: Alimentação: construção de um plano alimentar saudável com café da manhã, lanche, almoço, lanche e janta. Sono: ensinamento de exercício respiratório para alívio da ansiedade antes de dormir e exercício de reconhecimento de acontecimento positivo durante o dia. Atividade física: construção de um plano de atividade física periódico. Atividade de lazer: identificação de atividades de lazer para serem incorporadas às horas de descanso.

O Treino de Controle do Stress (TCS) é uma modalidade da terapia cognitivo-comportamental que tem como objetivo mudar hábitos de vida e de comportamento em quatro áreas que constroem os pilares do TCS: nutrição antiestresse, relaxamento da tensão mental e física, exercício físico e mudanças cognitivas comportamentais (Men, 2003). Ao final do encontro, foi realizado o fechamento, com o repasse dos principais aspectos vistos e a entrega, aos participantes, da tarefa de casa Reduza o estresse. Aprenda a dizer não (ou sim)! (Mathieu, 2012), que consiste em uma continuação da tarefa O que tem no seu prato? e busca, a partir das situações estressantes do dia a dia identificadas pelo participante, estimular o indivíduo a planejar-se e alterar determinados costumes de sua rotina que potencializam estas situações, buscando inserir hábitos saudáveis e atividades que as amenizem.

A Fadiga por Compaixão foi o tema da quarta sessão. Entretanto, antes de iniciá-la, deu-se a discussão da tarefa de casa e uma revisão dos assuntos vistos até o presente momento. Essa revisão é feita para reforçar, com os participantes, o entendimento dos tópicos tratados e das atividades realizadas até esse ponto da intervenção a fim de oportunizar o debate sobre o seu aproveitamento e impacto em sua vida profissional, bem como eventuais ajustes ou esclarecimentos que sejam necessários. Os participantes manifestaram os impactos gerados na sua rotina e em como a realização deste programa estava oportunizando a reflexão sobre certos hábitos de vida e promoção da saúde no trabalho.

Após essa reflexão, apresentou-se o tema Fadiga por Compaixão, que tem se apresentado, juntamente com o Burnout, como um problema de saúde que acomete principalmente profissionais que trabalham na linha de frente de emergências (Florio, 2010). A Fadiga por Compaixão é entendida como o resultado pelo consumo de altos níveis de energia e compaixão, durante um período prolongado de tempo, às pessoas que estão em sofrimento, muitas vezes, sem poder experimentar os resultados positivos de vê-los melhorarem (McHolm, 2006).

Introduziu-se a temática com uma atividade de comunicação realizada em dupla e trio, em que uma pessoa do grupo assume o papel de ouvinte e os demais de comunicadores. Os comunicadores escolhem uma história impactante para contar e os ouvintes são orientados pelo instrutor a assumirem dois tipos de ouvintes, um atento e um distraído. Ao final discutiu-se sobre os efeitos da história no ouvinte atento e no distraído, bem como as diferentes reações do comunicador. O objetivo desta atividade é proporcionar aos participantes o entendimento sobre empatia e compaixão, e como ela está afeta o dia a dia do profissional bombeiro.

Realizada a atividade foi apresentada a definição de Fadiga da Compaixão, suas diferenças em relação à $\mathrm{SB}$ e seus principais sintomas físicos, comportamentais e psicológicos. No término do encontro, realizou-se o 
fechamento do módulo com o repasse do tema abordado e entrega, aos participantes, da tarefa de casa. A atividade consistiu na realização de uma atividade de lazer que o participante havia colocado no seu Plano de Gerenciamento do Estresse.

Na quinta, e última, sessão, trabalhou-se o desenvolvimento de habilidades sociais, bem como realizou-se o fechamento da intervenção e avaliação final do trabalho. O Treinamento das Habilidades Sociais (THS) encontra-se entre as técnicas mais eficazes para melhorar a efetividade das condutas interpessoais e a qualidade de vida (Caballo, 2010), aspecto fundamental considerando que a SB tem em um dos seus principais componentes as relações interpessoais estabelecidas com a clientela atendida.

O comportamento considerado socialmente hábil é aquele que leva em consideração um conjunto de condutas, emitidas pelo indivíduo, que sejam adequadas à situação e ao contexto interpessoal que ele se encontra. Deve levar em consideração o comportamento dos demais e geralmente resolve problemas e conflitos imediatos, dessa forma, reduzindo a probabilidade de gerar problemas futuros (Caballo, 2010).

Para desenvolver o trabalho desse encontro, fez-se, inicialmente, a revisão da tarefa de casa, passada ao final do quarto encontro, para que, depois, fosse iniciado o tema HS. A primeira parte da sessão foi expositiva e consistiu na apresentação da definição, características e dimensões comportamentais da HS. Após, o encontro foi desenvolvido com base na atividade prática Ensaio do Comportamento. Este procedimento é frequentemente empregado nos THS, pois, por meio dele, representam-se maneiras apropriadas e efetivas de enfrentar situações que na vida real são problemáticas para os participantes. Seu objetivo consiste em aprender a modificar modos de respostas não adaptativas, substituindo-as por novas respostas (Caballo, 2010).

Para a atividade, os participantes foram divididos em uma dupla e um trio. Cada grupo, a partir dos conceitos e entendimentos expostos inicialmente, deveria criar duas situações geradoras de problemas, que caracterizassem seu dia a dia de trabalho, para encenar aos demais colegas. As encenações deveriam ser curtas, de no máximo 3 minutos cada. Ao final de cada apresentação, os participantes deveriam classificar o comportamento representado como assertivo - quando o comportamento é expresso de forma manifesta e sem exercer coação sobre a outra pessoa; não assertivo - quando há falta de expressão do comportamento, ou faz-se de forma indireta, sem intimidar o outro; agressivo - quando o comportamento é expresso de forma manifesta, mas de modo coercitivo sobre a outra pessoa; ou agressivo passivo - quando o comportamento é expresso de maneira indireta, coagindo a outra pessoa, tentando controlar o comportamento da outra pessoa de maneira indireta e sutil; e buscar, a partir desta mesma classificação, construir alternativas possíveis e eficazes que possam ser adotadas na situação encenada.

Encerrada a atividade, realizou-se um resumo sobre o tema trabalhado para que fosse dado início ao fechamento da intervenção. Após três meses de trabalho, concentrados em encontros quinzenais, os participantes desenvolveram conhecimentos e habilidades sobre os temas SB, Estratégias de Enfrentamento, Manejo de Estresse, Fadiga por Compaixão e Habilidades Sociais.

Assim, para encerramento da intervenção, foram repassados os temas tratados e o resumo dos entendimentos assimilados com o intuito de reforçar o conhecimento e/ou sanar possíveis dúvidas. Reforçou-se, então, a associação desses temas com sua prática profissional, buscando resgatar, especialmente, os aspectos geradores de satisfação na profissão e a importância de se estabelecer uma continuidade para as práticas de autocuidado desenvolvidas ao longo da intervenção, para que a profissão não seja uma fonte de adoecimento, mas sim de realização.

Foi reservado um espaço para a avaliação da intervenção. Neste momento, a coordenadora buscou, com os envolvidos, um feedback sobre aspectos positivos e oportunidades de melhorias da intervenção, como temáticas propostas, exercícios propostos e impacto no trabalho. Essa avaliação visa identificar aspectos que possam ser ajustados, para que intervenções futuras possam se beneficiar de tal experiência. O tempo final foi destinado para que os participantes respondessem aos questionários de Avaliação de Impacto do Treinamento no Trabalho (Abbad, 1999), que têm por objetivo identificar os efeitos gerados pela intervenção no comportamento dos participantes e as repercussões geradas em seu desempenho global, atitudes e motivação (Abbad, Gama, \& Borges-Andrade, 2000; Lacerda \& Abbad, 2003). Foram identificados os seguintes aspectos na avaliação: mudanças comportamentais e ganho de tranquilidade, aumento da espontaneidade e assertividade nas relações interpessoais e habilidades sociais, oportunidade de autoconhecimento, reconhecimento 
das limitações e como aprimorá-las e humanização do profissional. Como aspectos para melhorar e sugestões, foram relatados os seguintes pontos: poucos participantes, resistência dos colegas em participar, aumento da carga horária e inclusão da matéria no currículo de formação do profissional bombeiro.

\section{Considerações finais}

O estudo apresentou a proposta de um programa de intervenção de prevenção para manejo de estresse e SB a profissionais bombeiros. No que diz respeito a implicações para a prática, este relato contribui tanto para pesquisadores como para profissionais que trabaIham com ações de promoção e prevenção de saúde ocupacional, na medida em que apresenta ferramentas e técnicas que possibilitam aos trabalhadores lidarem melhor com os estressores laborais. Essa experiência mostrou-se relevante na medida em que proporcionou um espaço para a troca de experiências e debate sobre os fatores de risco, próprios da profissão, e formas de prevenção e promoção da saúde mental, uma vez que os conhecimentos puderam ser entendidos e debatidos a partir das vivências de seus participantes.

A participação de profissionais Sargentos, ocupantes de posição de chefia, permitiu instrumentaliza-los para lidarem com questões relacionadas ao manejo do estresse e SB, o que para muitos gestores é um grande desafio a ser vencido. Tornando-os capazes de desenvolver o potencial de trabalho de toda sua equipe, fazendo com que essa se sinta capaz de transformar e realizar com sucesso todos os projetos desenvolvidos pela instituição, atentando-se às queixas relativas à saúde ocupacional.

Algumas questões devem ser consideradas para o planejamento de futuras intervenções. A primeira refere-se ao número reduzido de participantes, pois é possível que profissionais acometidos ou mais suscetíveis ao Burnout não tenham participado da intervenção. Os profissionais acometidos pelo Burnout podem entender esse espaço como mais uma atividade a ser realizada após o período de trabalho, configurando-se como um fator de estresse. Neste sentido, sugere-se um maior período de divulgação e a incorporação da intervenção a atividades formais na organização como treinamentos técnicos obrigatórios.

Além disso, o número reduzido de sessões mostra-se menos eficiente quando comparado aos programas de longa duração. Uma comparação entre propostas de intervenção direcionadas aos indivíduos mostra que impactos importantes nos níveis de Burnout costumam aparecer em programas com mais de seis meses de duração (Awa, Plaumann, \& Walter, 2010). Desta forma, recomenda-se a ampliação do número de sessões para 10, com cinco meses de duração, bem como a realização de reforços periódicos, com o propósito de realizar reciclagens de conhecimento. Essa medida tem se mostrado positiva, resultando em efeitos mais duradouros de redução de níveis de Burnout nos programas de intervenção que adotaram essa prática (Awa, Plaumann, \& Walter, 2010). Os estudos de delineamento longitudinais permitem maior acompanhamento e realização de medições recorrentes, dessa forma, contribuindo para ampliar a compreensão sobre o modelo de Burnout, bem como para ajudar a explorar os seus antecedentes.

\section{Referências}

Abalo, J. A. G., \& Roger, M. C. (1998, agosto). Burnout: una amenaza a los equipo de la salud. Conferência apresentada em II Jornada de Actualización en Psicología de la Salud - Asociación Colombiana de Psicologia de la Salud - ASOCOPSIS, Cali, Colômbia.

Abbad, G. (1999). Um modelo integrado de avaliação do impacto do treinamento no trabalho - IMPACT (Tese de doutorado não-publicada). Universidade de Brasília. Brasília.

Abbad, G. S., Gama, A. L. G., \& Borges-Andrade, J. E. (2000) Treinamento: análise do relacionamento da avaliação nos níveis de reação, aprendizagem e impacto no trabalho. Revista de Administração Contemporânea, 4(3), 25-45. doi: 10.1590/ S1415-65552000000300003

Awa, W. L., Plaumann, M., \& Walter, U. (2010). Burnout prevention: a review of intervention programs. Patient Education and Counseling, 78(2), 184-190. doi:10.1016/j.pec.2009.04.008

Brock, B. L., \& Grady, M. L. (2000). Rekindling the flame: principals combating teacher burnout. Thousand Oaks: Corwin Press.

Byrne, B. M. (1993). The Maslach Burnout Inventory: testing for factorial validity and invariance across elementary, intermediate and secondary teachers. Journal of Occupational and Organizational Psychology, 66(3), 197-213. doi: 10.1111/j.2044-8325.1993. tb00532.x

Caballo, V. E. (2010). Manual de avaliação e treinamento das habilidades sociais. São Paulo: Santos.

Carlotto, M. S. (2012). Síndrome de Burnout em professores: avaliação, fatores associados e intervenção. Porto: LivPsic.

Carlotto, M. S., \& Gobbi, M. D. (1999). Síndrome de burnout: um problema do indivíduo ou do seu contexto de trabalho? Aletheia, 10, 103-114.

Cebrià-Andreu, J. (2005). El síndrome de desgaste profesional como problema de salud pública. Gaceta Sanitária, 19(6), 463-470. Recuperado de http://scielo.isciii.es/scielo. php?script=sci_arttext\&pid=S0213-91112005000600008\&Ing= es\&tlng=es 
Dierendonck, D. van, Schaufeli, W. B., \& Buunk, B. P. (1998). The evaluation of an individual burnout intervention program: the role of inequity and social support. Journal of Applied Psychology, 83(3), 392407. doi:10.1037/0021-9010.83.3.392

Florio, C. (2010). Burnout and compassion fatigue: a guide for mental health professionals and care givers. Lexington: MSW, LPC, LADC.

Folkman, S., \& Lazarus, R. S. (1980). An analysis of coping in a middleaged community sample. Journal of Health and Social Behavior, 21(3), 219-239. Recuperado de http://www.jstor.org/stable/2136617

Gil-Monte, P. R. (2005). El síndrome de quemarse por el trabajo (burnout). Una enfermedad laboral en la sociedad del bienestar. Madrid: Pirâmide.

Gunasingam, N., Burns, K, Edwards, J., Dinh, M., \& Walton, M. (2015). Reducing stress and burnout in junior doctors: the impact of debriefing sessions. Postgraduate Medical Journal, 91(1074), 182-187. doi:10.1136/postgradmedj-2014-132847

Halbesleben, J. R. B., \& Buckley, M. R. (2004). Burnout in organizational life. Journal of Management, 30(6), 859-879.

Halbesleben, J. R. B., Osburn, H. K., \& Mumford, M. D. (2006). Action research as a burnout intervention: Reducing burnout in the federal fire service. The Journal of Applied Behavioral Science, 42(2), 244266. doi: $10.1177 / 0021886305285031$

Harrison, B. J. (1999). Are you destined to burn out? Fund Raising Management, 30(3), 25-27.

Kyriacou, C., \& Sutcliffe, J. (1978). Teacher stress: prevalence, sources and symptoms. Educational Psychology, 48(2), 159-16. doi: 10.1111/j.2044-8279.1978.tb02381.x

Lacerda, É. R. M., \& Abbad, G. (2003). Impacto do treinamento no trabalho: investigando variáveis motivacionais e organizacionais como suas preditoras. Revista de Administração Comtemporânea, 7(4), 77-96. doi: 10.1590/S1415-65552003000400005

Lazarus, R. L., \& Folkman, S. (1984). Stress, appraisal and coping. Nova lorque: Springer.

Leiter, M. P., Bakker, A. B., \& Maslach, C. (2014). Burnout at work: a psychological perspective. Nova lorque, NY: Psychology Press

Maslach, C., \& Jackson, S. E. (1981). The measurement of experienced burnout. Journal of Organizational Behavior, 2(2), 99-113. doi: 10.1002/job.4030020205

Maslach, C., \& Leiter, M. P. (2008). Early predictors of job burnout and engagement. Journal of Applied Psychology, 93(3), 498-512. doi: 10.1037/0021-9010.93.3.498

Maslach, C., \& Schaufeli, W. B. (1993). Historical and conceptual development of burnout. In W. B. Schaufeli, C. Maslach, \& T. Marek (Orgs.), Professional burnout: recent developments in theory and research (pp. 1-16). Philadelphia: Taylor \& Francis.

Maslach, C., Schaufeli, W. B., \& Leiter, M. P. (2001). Job burnout. Annual Review of Psychology, 52, 397-422. doi: 10.1146/annurev. psych.52.1.397
Mathieu, F. (2012). The compassion fatigue workbook. Nova lorque: Routledge.

McHolm, F. (2006). Rx for compassion fatigue. Journal of Chistian Nursing, 23(4), 12-19.

Men, L. (2003). Mecanismos neuropsicofisiológicos do stress: teoria e aplicações clínicas. São Paulo: Casa do Psicólogo.

Mirvis, D. M., Graney, M. J., Ingram, L., Tang, J., \& Kilpatrick, A. O (2006). Burnout and psychological stress among deans of colleges of medicine: a national study. Journal of Health and Human Services Administration, 29(1), 4-25. Recuperado de http://www. jstor.org/stable/25790676

Murphy, L. R. (1996). Stress management in work settings: a critical review of the health effects. American Journal of Health Promotion, 11(2), 112-135. doi:10.4278/0890-1171-11.2.112

Murta, S. G., Laros, J. A., \& Tróccoli, B. T. (2005). Manejo de estresse ocupacional na perspectiva da área de avaliação de programas. Estudos de Psicologia, 10(2), 167-176. doi: 10.1590/ S1413-294X2005000200002

Murta, S. G., \& Tróccoli, B. T. (2007). Stress ocupacional em bombeiros: efeitos de intervenção baseada em avaliação de necessidades. Estudos de Psicologia, 24(1), 410-51. doi: 10.1590/ S0103-166X2007000100005

Neves, E. P., \& Wink, S. (2007). O autocuidado no processo de viver: enfermeiras compartilham concepções e vivências em sua trajetória profissional. Texto\& Contexto - Enfermagem, 16(1), 172-179. doi: 10.1590/S0104-07072007000100023

Resolução no 466, de 12 de dezembro de 2012 (2012). Diretrizes e normas regulamentadoras de pesquisas envolvendo seres humanos. Brasília: Ministério da Saúde.

Rowe, M. M. (2000). Skills training in the long-term management of stress and occupational burnout. Current Psychology, 19(3), 215228. doi: 10.1007/s12144-000-1016-6

Salanova, M., \& Llorens, S. (2008). Estado actual y retos futuros en el estudio del burnout. Papeles del Psicólogo, 29(1), 59-67. Recuperado de http://www.papelesdelpsicologo.es/ contenido?num $=1126$

Soares, J. I. S., \& Rocha, J. C. (2010). Exaustão emocional dos cuidadores dos doentes oncológicos: eficácia de uma intervenção de grupo (Tese de doutorado não publicada). Universidade do Porto, Portugal.

Trigo, T. R., Teng, C. T., \& Hallak, J. E. C. (2007). Síndrome de burnout ou estafa profissional e os transtornos psiquiátricos. Revista de Psiquiatria Clínica, 34(5), 223-233. doi: 10.1590/ S0101-60832007000500004

Van der Klink, J. J., Blonk, R. W., Schene, A. H., \& Van Dijk, F. J. (2001). The benefits of interventions for work-related stress. American Journal of Public Health, 91(2), 270-276. doi:10.2105/ ajph.91.2.270 
Programa de prevenção para manejo de estresse e Síndrome de Burnout para bombeiros: Relato de experiência de uma intervenção

Lucia Petrucci de Melo, Mestre em Psicologia Social pela Pontifícia Universidade Católica do Rio Grande do Sul (PUCRS), é Gerente de

Recursos Humanos do Sheraton Hotel Porto Alegre. Endereço para correspondência: Rua Heretiano Rocha 76/404, Petrópolis, Porto Alegre/RS, Brasil. Telefone (51)99913-2358. E-mail: lulucitamelo@hotmail.com

Mary Sandra Carlotto, Doutorado pela Universidade de Santiago de Compostela (USC), Espanha, é Docente do Programa de Pós Graduação em Psicologia da Universidade do Vale do Rio dos Sinos (UNISINOS). E-mail: mscarlotto@pesquisador.cnpq.br

Recebido em 09.Dez.15 Revisado em 17.Ago.16 Aceito em 06.Mar.17 